\title{
A new approach to bulk viscosity in strange quark matter at high densities
}

\author{
Shou-wan Chen ${ }^{1}$, Hui Dong ${ }^{2}$, and Qun Wang ${ }^{1}$ \\ ${ }^{1}$ Department of Modern Physics, University of Science and Technology of China, \\ Hefei 230026, P.R.China \\ ${ }^{2}$ School of Physics, Shandong University, Jinan 250100, P.R.China \\ E-mail: windwan@mail.ustc.edu.cn, hdong@sdu.edu.cn, qunwang@ustc.edu.cn
}

\begin{abstract}
A new method is proposed to compute the bulk viscosity in strange quark matter at high densities. Using the method it is straightforward to prove that the bulk viscosity is positive definite, which is not so easy to accomplish in other approaches especially for multi-component fluids like strange quark matter with light up and down quarks and massive strange quarks.
\end{abstract}


There are a lot of works in literature concerning the bulk viscosity for quark and nuclear matter in compact stars. Some are based on the Urca and non-leptonic weak processes for nucleons and hyperons [1, 2, 13, 5, 4, 6, 17, 8, 9, 11, 10, 12, 13, 14, 15, 16, 17, 18. Others are about Urca processes and $d-s$ transitions for unpaired quark matter [19, 20, 21, 22, 23, 24, 25] and some color superconducting phases [26, 27, 28]. For a brief review, see e.g. Ref. [29]. In the CFL phase the bulk viscosity comes from the lightest degrees of freedom, e.g. kaons, superfluid $H$ modes etc., which are no longer quarks [30, 31, 32]. In this note, we will only consider strange quark matter in normal state, for recent reviews of strange quark matter in stars, see e.g. [33, 34]. It is believed that in the regime of high density far above the saturation one of nuclear matter, the degrees of freedom become quarks and gluons. At weak coupling limit for quark matter one can make reliable predictions based on perturbative quantum chromodynamics. The bulk viscosity in compact star environment is partly due to damping mechanism for external perturbations which can lead to instabilities such as r-mode instability [35, 36, 37, 38, 39, 40]. The stars can drastically spin down by gravitational radiation when the bulk viscosity is insufficient.

Bulk viscosity arises from energy dissipation due to the change of fluid volume. The fluid will deviate from thermal and/or chemical equilibrium when it is compressed or rarefied. The physical pressure (i.e. the instantaneous one) will differ from its equilibrium value given by the equation of state at the same energy density. Energy has to be consumed to re-equilibrate the system. The bulk viscosity, denoted by $\zeta$, is defined through the rate of energy dissipation,

$$
\left\langle\dot{\varepsilon}_{\mathrm{diss}}\right\rangle=\frac{\zeta}{\tau} \int_{0}^{\tau}(\nabla \cdot \mathbf{v})^{2} d t=-\frac{n}{\tau} \int_{0}^{\tau} P \dot{V} d t
$$

where $\tau=2 \pi / \omega$ represents the pulsation period, $\nabla \cdot \mathbf{v}$ the divergence of fluid velocity, $n$ the number density, $P$ the physical pressure, and $V=1 / n$ the specific volume.

The bulk viscosity can be defined in an alternative way in terms of complex variables. The deviation of the physical pressure, $P$, from the corresponding equilibrium one, $P_{\text {eq }}$, can be related to the bulk viscosity in the form,

$$
\delta P=P-P_{\text {eq }}=-\zeta_{\mathrm{c}} \nabla \cdot \mathbf{v},
$$

where $\zeta_{\mathrm{c}}$ is complex. The physical bulk viscosity is then given by the real part $\Re\left(\zeta_{\mathrm{c}}\right)$.

There are two characteristic time scales related to bulk viscosity, one corresponds to perturbation and the other to relaxation. The dominant contribution to dissipative energy loss comes from the re-equilibration whose time scale is comparable to that of external perturbations. For example, in strange quark matter which consists of up and down quarks which are massless, strange quarks which are massive and electrons, the time scale of the weak interaction in the system is comparable to the typical one for pulsations induced by external perturbations. To calculate the bulk viscosity, we can only consider weak processes.

In literature about the bulk viscosity for quark or nuclear matter, the simple fluid picture is always implied. In the real world, however, the strange quark mass is not 
negligible. For those external perturbations which do not distinguish particle species, the pressures felt by different ingredients are the same. This would lead to equal momentum variations but different velocity ones due to different masses. Although the time scale for strong interaction through which the quarks of different flavors reach thermal equilibrium is much shorter than that of weak interaction responsible for the bulk viscosity, there are no constraints about fluid velocities of different species either from hydrodynamic equations or from particle distribution functions, because the fluid velocity is the thermal average of particle microscopic velocities, which vary widely for particles with different masses even when they have the same temperature. Especially the divergences of the fluid velocities for particles with different masses are not necessary the same due to the difference in equations of state, since we have $\nabla \cdot \mathbf{v}=-\frac{1}{\epsilon+P} \frac{\partial \epsilon}{\partial t}$ from the fluid equation in the local co-moving frame, where the energy density $\epsilon$ and the pressure $P$ depend on masses. Therefore there is no strong reason that strange quark matter should be regarded as a simple fluid if the strange quark mass is large compared to temperature and chemical potential, i.e. the simple fluid picture for strange quark matter is just an approximation when the strange quark mass is very large. A natural question to ask is: whether conventional approaches in literature which are successfully applied to the simple fluid picture are still valid for multi-component fluids.

Based on above arguments we extended conventional approaches to strange quark matter as a multi-component fluid. We obtained general constraints on fluid velocity divergences for different particle species enforced by baryon number conservation and electric charge neutrality. We also found a new oscillation pattern which gives a new solution to the bulk viscosity in the multi-component fluid satisfying these constraints. The resulting bulk viscosity is about one order of magnitude larger than those from conventional approaches. Our further investigations indicate that the positivity of the results in our previous paper [25] can not always be guaranteed for some cases although allowed by those constraints. In this note we propose an alternative way of computing the bulk viscosity of strange quark matter as a multi-component fluid in terms of entropy production, where the bulk viscosity is definitely positive. The organization of the note is as follows. First we summarize the formalism to calculate the bulk viscosity for strange quark matter. Then we introduce our new approach to bulk viscosity by relating the energy dissipation to entropy production.

In strange quark matter the weak processes via the exchange of $W^{ \pm}$bosons relevant to bulk viscosity are

$$
\begin{array}{ll}
\text { 1. } & u+d \leftrightarrow u+s, \quad(\mathrm{~d} \leftrightarrow \mathrm{s} \text { transition }), \\
\text { 2. } & u+e \leftrightarrow d+\nu, \quad(\text { Urca } \mathrm{I}), \\
\text { 3. } & u+e \leftrightarrow s+\nu, \quad(\text { Urca II). }
\end{array}
$$

Note that the reverse reactions in the second and third lines (Urca I and II) are not real ones but represent $d / s \rightarrow u+e+\bar{\nu}$. In thermal and chemical equilibrium all chemical potentials satisfy,

$$
\mu_{d} \quad=\mu_{s}
$$




$$
\begin{aligned}
& \mu_{u}+\mu_{e}=\mu_{d}, \\
& \mu_{u}+\mu_{e}=\mu_{s} .
\end{aligned}
$$

Considering there is no accumulation of neutrinos in the system, we have set $\mu_{\nu / \bar{\nu}}=0$ because neutrino mean free paths are much longer than typical radii of compact stars. The local charge neutrality also plays an important role,

$$
\sum_{i=u, d, s} Q_{i} n_{i}-n_{e}=0
$$

where $Q_{i}$ are electric charges in unit of $e$. From Eqs. (44) and (5) the equilibrium values of chemical potentials and number densities can be obtained.

Volume oscillations induced by perturbations lead to chemical non-equilibrium, since the chemical potential is related to the number density $n_{i}$ for quark species $i$ through the equation of state. The baryon number density is then $n_{B} \equiv \sum_{i} n_{i} / 3$. We can use the oscillation of the baryon number density, $\delta n_{B}=\delta n_{B 0} e^{i \omega t}$, to account for the volume oscillation. Hereafter we will keep the subscript $i$ for quarks and $j$ for quarks and electrons. Due to local charge neutrality, the density oscillations $\delta n_{j}$ are not independent from each other. The chemical potential differences between two sides of reactions are written as

$$
\begin{aligned}
& \Delta \mu_{1} \equiv \mu_{s}-\mu_{d}=\delta \mu_{s}-\delta \mu_{d} \\
& \Delta \mu_{2} \equiv \mu_{d}-\mu_{u}-\mu_{e}=\delta \mu_{d}-\delta \mu_{u}-\delta \mu_{e}, \\
& \Delta \mu_{3} \equiv \mu_{s}-\mu_{u}-\mu_{e}=\delta \mu_{s}-\delta \mu_{u}-\delta \mu_{e} .
\end{aligned}
$$

The above equations can be put into more compact form, $\Delta \mu_{k}=c_{k j} \delta \mu_{j}$. Here $c_{k j}$ ( $k=1,2,3$ denote different reactions) are coefficients in front of chemical potentials in (6). The reaction rates are proportional to these differences of chemical potentials when $\Delta \mu_{k} \ll T(\ll \mu)$,

$$
\Gamma_{k}=\lambda_{k} \Delta \mu_{k}\left(=\lambda_{k} c_{k j} \delta \mu_{j}\right) .
$$

The coefficients $\lambda_{k}$ can be obtained from Boltzmann equation with collision terms.

For each ingredient in strange quark matter continuity equations read

$$
\dot{n}_{j}+n_{j} \boldsymbol{\nabla} \cdot \mathbf{v}_{j}=J_{j} .
$$

The source terms in the right-hand-side can be expressed in a linear combination of $\Gamma_{k}$,

$$
J_{j}=-c_{k j} \Gamma_{k}=-c_{k j} \lambda_{k} c_{k j^{\prime}} \delta \mu_{j^{\prime}} .
$$

Note that there is no source term for baryon number since it is conserved in all processes. So we have

$$
\dot{n}_{B}+n_{B} \boldsymbol{\nabla} \cdot \mathbf{v}_{B}=0 .
$$

The conservation of the baryon number and the electric charge in all three reactions lead to relations about sources,

$$
\begin{aligned}
& \frac{1}{3} \sum_{i} J_{i}=0, \\
& \sum_{i} Q_{i} J_{i}-J_{e}=0 .
\end{aligned}
$$


Substituting above relations into continuity equations (8) and (10), we obtain general constraints for fluid velocity divergences,

$$
\begin{aligned}
& n_{B} \boldsymbol{\nabla} \cdot \mathbf{v}_{B}=\sum_{i} \frac{1}{3} n_{i} \boldsymbol{\nabla} \cdot \mathbf{v}_{i} \\
& n_{e} \boldsymbol{\nabla} \cdot \mathbf{v}_{e}=\sum_{i} Q_{i} n_{i} \boldsymbol{\nabla} \cdot \mathbf{v}_{i} .
\end{aligned}
$$

To solve $n_{j}$ additional inputs are required. In traditional treatments $n_{B} \dot{X}_{j}=J_{j}$ is used implicitly, where $X_{j} \equiv n_{j} / n_{B}$ is the number density fraction of baryons for particle $j$. It can easily be verified from the general constraints (11) that the usage of $n_{B} \dot{X}_{j}=J_{j}$ implies $\nabla \cdot \mathbf{v}_{j}=\nabla \cdot \mathbf{v}_{B}$ for all particle species. This holds only if the mass differences among different particle species are negligible. However the strange quark mass $m_{s}$ could be much larger than light quark ones. So the fluid velocity of the strange component could differ from that of the light sector. In our previous paper [25] we considered a limit case where $\nabla \cdot \mathbf{v}_{s}=0$ and $\nabla \cdot \mathbf{v}_{u}=\nabla \cdot \mathbf{v}_{d}$, which provides additional conditions to fix $n_{j}$. The numerical results are about one order of magnitude larger than those from traditional solutions [19, 20, 21, 22, 23, 24].

Now we turn to the issue about the positivity of the bulk viscosity. We will provide an explicit and general demonstration about the positivity regardless of whether the system is a simple fluid or multi-component fluid. The angle in which we look at the problem is the possible connection between the energy dissipation and the entropy production related to bulk viscosity arising from irreversibility of the periodic compression-expansion process. Note that in an irreversible process the entropy of the system always increases.

To observe the entropy change we separate the fluid into small volume cells and focus on one of them. Since the fluid velocity divergences for particles with different masses are not identical, or the corresponding hydrodynamic motions are different, the cell is not a closed system and has exchange of particles with others. Due to baryon number conservation we can choose the cell in the way that the total baryon number in the cell does not change. It is an open system but with a global control number, so we can call it quasi-control number system. We assume that the quasi-equilibrium is reached in the cell, i.e. the system is in full thermodynamic equilibrium except the chemical one, since the chemical reactions from weak interaction proceed much slower than strong processes. The entropy of the cell increases during the chemical reequilibration. Neglecting the exchange of particles and heat on the surface of the whole system with outside, the net change of particles and heat vanishes. Therefore after one cycle of compression-expansion process, the only thermodynamic quantity that changes is the entropy. We also neglect temperature change as in traditional treatments. The change of internal energy in one period reads

$$
U(t+\tau)-U(t)=\int_{\tau} \mathrm{d} t \frac{\mathrm{d}}{\mathrm{d} t} \sum_{l} T s_{N}^{\mathrm{i}} N_{B}^{l}=N_{B} \int_{\tau} \frac{T \mathrm{~d} s_{N}^{\mathrm{i}}}{\mathrm{d} t} d t,
$$

where the superscript $l$ represents the local cell, $N_{B}^{l}$ its baryon number, and $s_{N}^{\mathrm{i}}$ the 
entropy per baryon number due to internal irreversible processes. Using the formula for entropy production in dissipative hydrodynamics we obtain,

$$
\frac{T \mathrm{~d} s_{N}^{\mathrm{i}}}{\mathrm{d} t}=-\mu_{j} \frac{\mathrm{d} X_{j}^{\mathrm{i}}}{\mathrm{d} t}=\frac{1}{n_{B} \lambda_{k}\left(\Delta \mu_{k}\right)^{2}}
$$

Then the second type of definition for bulk viscosity becomes

$$
\frac{\zeta}{n_{B}} \int_{\tau}\left(\boldsymbol{\nabla} \cdot \mathbf{v}_{B}\right)^{2} \mathrm{~d} t=\int_{\tau} \frac{1}{n_{B}} \lambda_{k}(\Delta \mu)_{k}^{2} \mathrm{~d} t .
$$

Finally we get the positive bulk viscosity in the new approach,

$$
\zeta=\frac{\int_{\tau} \lambda_{k}(\Delta \mu)_{k}^{2} \mathrm{~d} t}{\int_{\tau}\left(\boldsymbol{\nabla} \cdot \mathbf{v}_{B}\right)^{2} \mathrm{~d} t} .
$$

It can be proved that the above formula can reproduce the bulk viscosity for the simple (single-component) fluids.

In summary, from the connection between the dissipative energy loss and the entropy production a new approach to calculating bulk viscosity in strange quark matter has been developed. The positivity of the bulk viscosity can easily be observed in the approach for simple and multi-component fluids.

Acknowledgement. Q.W. is supported in part by '100 talents' project of Chinese Academy of Sciences (CAS), by National Natural Science Foundation of China (NSFC) under the grants 10675109 and 10735040 . H.D. is supported by National Natural Science Foundation of China (NSFC) under the grant 10847149.

[1] W. D. Langer and A. G. W. Cameron, Astrophys. Space Sci. 5, 213 (1969).

[2] P. B. Jones, Proc. R. Soc. A 323, 111 (1971).

[3] R. F. Sawyer, Phys. Rev. D 39, 3804 (1989).

[4] M. Prakash, M. Prakash, J. M. Lattimer and C. J. Pethick, Astrophys. J. 390, L77 (1992).

[5] P. Haensel and R. Schaeffer, Phys. Rev. D 45, 4708 (1992).

[6] P. Haensel, K. P. Levenfish and D. G. Yakovlev, Astron. Astrophys. 357, 1157 (2000) arXiv:astro-ph/0004183.

[7] P. Haensel, K. P. Levenfish and D. G. Yakovlev, Astron. Astrophys. 372, 130 (2001) arXiv:astro-ph/0103290.

[8] P. B. Jones, Phys. Rev. Lett. 86, 1384 (2001).

[9] P. B. Jones, Phys. Rev. D 64, 084003 (2001).

[10] L. Lindblom and B. J. Owen, Phys. Rev. D 65, 063006 (2002) arXiv:astro-ph/0110558.

[11] P. Haensel, K. P. Levenfish and D. G. Yakovlev, Astron. Astrophys. 381, 1080 (2002) arXiv:astro-ph/0110575.

[12] E. N. E. van Dalen and A. E. L. Dieperink, Phys. Rev. C 69, 025802 (2004) arXiv:nucl-th/0311103.

[13] M. Nayyar and B. J. Owen, Phys. Rev. D 73, 084001 (2006) arXiv:astro-ph/0512041].

[14] D. Chatterjee and D. Bandyopadhyay, Phys. Rev. D 74, 023003 (2006) arXiv:astro-ph/0602538.

[15] D. Chatterjee and D. Bandyopadhyay, Phys. Rev. D 75, 123006 (2007) arXiv:astro-ph/0702259.

[16] M. E. Gusakov, Phys. Rev. D 76, 083001 (2007) arXiv:0704.1071 [astro-ph]].

[17] D. Chatterjee and D. Bandyopadhyay, arXiv:0712.3171 [astro-ph].

[18] D. Chatterjee and D. Bandyopadhyay, arXiv:0712.4347 [astro-ph].

[19] Q. D. Wang and T. Lu, Phys. Lett. B 148, 211 (1984).

[20] R. F. Sawyer, Phys. Lett. B 233, 412 (1989) [Erratum-ibid. B 237, 605 (1990)].

[21] J. Madsen, Phys. Rev. D 46, 3290 (1992). 
[22] Z. G. Dai and T. Lu, Z. Phys. A 355, 415 (1996).

[23] J. D. Anand, N. Chandrika Devi, V. K. Gupta and S. Singh, Pramana 54, 737 (2000).

[24] X. P. Zheng, X. W. Liu, M. Kang and S. H. Yang, Phys. Rev. C 70, 015803 (2004).

[25] H. Dong, N. Su and Q. Wang, Phys. Rev. D 75, 074016 (2007) arXiv:astro-ph/0702104.

[26] B. A. Sa'd, I. A. Shovkovy and D. H. Rischke, Phys. Rev. D 75, 065016 (2007) arXiv:astro-ph/0607643.

[27] M. G. Alford and A. Schmitt, J. Phys. G 34, 67 (2007) arXiv:nucl-th/0608019.

[28] B. A. Sa'd, I. A. Shovkovy and D. H. Rischke, Phys. Rev. D 75, 125004 (2007) arXiv:astro-ph/0703016.

[29] H. Dong, N. Su and Q. Wang, J. Phys. G 34, S643 (2007) arXiv:astro-ph/0702181.

[30] M. G. Alford, M. Braby, S. Reddy and T. Schaefer, Phys. Rev. C 75, 055209 (2007) arXiv:nucl-th/0701067.

[31] C. Manuel and F. Llanes-Estrada, JCAP 0708, 001 (2007) [arXiv:0705.3909 [hep-ph]].

[32] M. G. Alford, M. Braby and A. Schmitt, J. Phys. G 35, 115007 (2008) arXiv:0806.0285 [nucl-th]].

[33] J. Schaffner-Bielich, J. Phys. G 31, S651 (2005) arXiv:astro-ph/0412215.

[34] J. Schaffner-Bielich, S. Schramm and H. Stocker, arXiv:0711.2639 [astro-ph].

[35] N. Andersson, Astrophys. J. 502, 708 (1998) arXiv:gr-qc/9706075.

[36] J. L. Friedman and S. M. Morsink, Astrophys. J. 502, 714 (1998) arXiv:gr-qc/9706073.

[37] L. Lindblom, B. J. Owen and S. M. Morsink, Phys. Rev. Lett. 80, 4843 (1998) arXiv:gr-qc/9803053.

[38] D. Lai, arXiv:astro-ph/0101042.

[39] M. Mannarelli, C. Manuel and B. A. Sa'd, Phys. Rev. Lett. 101, 241101 (2008) arXiv:0807.3264 [hep-ph]].

[40] W. j. Fu, H. q. Wei and Y. x. Liu, Phys. Rev. Lett. 101, 181102 (2008) [arXiv:0810.1084 [nucl-th]]. 